\title{
Quasi regular modules and trivial extension
}

\author{
Chillumuntala Jayaram ${ }^{1}$ (D), Ünsal Tekir*2 (D), Suat Koç ${ }^{2}$ (D) \\ ${ }^{1}$ The University of the West Indies, Department of CMP, P.O. Box 64, Bridgetown, Barbados \\ ${ }^{2}$ Marmara University, Department of Mathematics, Ziverbey, Goztepe, 34722, Istanbul, Turkey
}

\begin{abstract}
Recall that a ring $R$ is said to be a quasi regular ring if its total quotient ring $q(R)$ is von Neumann regular. It is well known that a ring $R$ is quasi regular if and only if it is a reduced ring satisfying the property: for each $a \in R$, $a n n_{R}\left(a n n_{R}(a)\right)=a n n_{R}(b)$ for some $b \in R$. Here, in this study, we extend the notion of quasi regular rings and rings which satisfy the aforementioned property to modules. We give many characterizations and properties of these two classes of modules. Moreover, we investigate the (weak) quasi regular property of trivial extension.
\end{abstract}

Mathematics Subject Classification (2020). 16E50, 13A15

Keywords. von Neumann regular ring, quasi regular ring, von Neumann regular module, quasi regular module, trivial extension

\section{Introduction}

In this paper, all rings are assumed to be commutative with $1 \neq 0$ and all modules are nonzero unital. Let $R$ always denote such a ring and $M$ always denote such an $R$-module. The concept of von Neumann regular rings has an important place in commutative algebra. There have been many generalizations and applications of von Neumann regular rings to other areas such as graph theory. See, for example, [2] and [10]. Previously, recall that a ring $R$ is said to be a von Neumann regular (for short, vn-regular) ring if for each $x \in R, x=x^{2} y$ for some $y \in R$ [14]. Note that a ring $R$ is vn-regular if and only if for each $x \in R,(x)=(e)$ for some idempotent element $e \in R$, where $(x)$ is the principal ideal generated by $x \in R$ if and only if it is a reduced and zero dimensional ring, i.e, every prime ideal is maximal if and only if the localization $R_{P}$ of $R$ at $P$ is a field for each prime ideal $P$ of $R$. Jayaram and Tekir extend the notion of vn-regular rings to modules in terms of $M$-regular elements [8]. Let $M$ be an $R$-module. Then $e \in R$ is said to be an $M$-regular (resp., a weak idempotent) element if $e M=e^{2} M$ (resp., $e m=e^{2} m$ for each $m \in M)$. Note that all idempotent elements are weak idempotent and these concepts are equal when $M$ is a faithful module. $M$ is called a vn-regular $R$-module if for each $m \in M$, there is an $e \in R$ such that $R m=e M=e^{2} M$ [8]. It is well known that a finitely

\footnotetext{
*Corresponding Author.

Email addresses: jayaram.chillumu@cavehill.uwi.edu (C. Jayaram), utekir@marmara.edu.tr (Ü. Tekir), suat.koc@marmara.edu.tr (S. Koç)

Received: 30.08.2019; Accepted: 04.05.2020
} 
generated $R$-module $M$ is a vn-regular module if and only if for each $m \in M$, there is a weak idempotent element $e \in R$ such that $R m=e M$ [8, Lemma 5].

One of the generalization of vn-regular rings is quasi regular (sometimes called complemented) rings. A ring $R$ is called a quasi regular ring if its total quotient ring $q(R)$ is a vn-regular ring. In [4, Theorem 2.2], it was shown that a ring $R$ is a quasi regular ring if and only if $R$ is a reduced ring and satisfies the condition: for each $a \in R$, $a n n_{R}\left(a n n_{R}(a)\right)=$ $a_{n n}(b)$ for some $b \in R$, where $a_{n n}(a)=\{x \in R: x a=0\}$. Here, we call a ring $R$ weak quasi regular (for short, wq-regular) if for each $a \in R, a \operatorname{ann}_{R}\left(a n n_{R}(a)\right)=a n n_{R}(b)$ for some $b \in R$. Note that all quasi regular rings are wq-regular. But the converse is not true: just consider a non-reduced principal ideal ring. For instance, $\mathbb{Z}_{4}$ is a wq-regular ring, but is not a quasi regular ring.

Our aim in this article is to extend the notion of quasi regular rings and wq-regular rings to modules. For the sake of thoroughness we give some definitions which we will need throughout this study. For each submodules $N$ and $K$ of $M$, the residual of $N$ by $K$ is defined by $\left(N:_{R} K\right)=\{r \in R: r K \subseteq N\}$. In particular, if $N=0$, we use $a n n_{R}(K)$ to denote $\left(0:_{R} K\right)$. Also for each cyclic submodule $R m$, we use $a n n_{R}(m)$ instead of $\operatorname{ann}_{R}(R m)$. Similarly, for each ideal $J$ of $R$ and each submodule $K$ of $N$, one can define residual of $N$ by $J$ as $(N: M J)=\{m \in M: J m \subseteq N\}$. In case $N=0$, we will use $\operatorname{ann}_{M}(J)$ instead of $\left(0:_{M} J\right)$ and also for each $a \in R$, we denote $\operatorname{ann}_{M}(R a)$ by $\operatorname{ann}_{M}(a)$.

Also the set $Z(M)$ of zero divisors on $M$ and the set $T(M)$ of all torsion elements of $M$ are defined as follows:

$$
\begin{aligned}
& Z(M)=\left\{a \in R: \operatorname{ann}_{M}(a) \neq 0\right\} \text { and } \\
& T(M)=\left\{m \in M: \operatorname{ann}_{R}(m) \neq 0\right\} .
\end{aligned}
$$

Note that $T(M)$ is not always a submodule of $M$ and similarly $Z(M)$ may not be an ideal of $R$. $M$ is called a torsion free module if $T(M)=0$. Also if $T(M)=M$, then $M$ is called a torsion module. Otherwise, we call that $M$ is a non-torsion module. Assume that $S=R-Z(M)$. It is easily seen that $S$ is a multiplicatively closed subset (briefly m.c.s) of $R$. Also the localization $M_{S}$ is an $R_{S}$-module and it is called the total quotient module of $M$. We denote the total quotient module by $q(M)$. We call that $M$ is a quasi regular $R$-module if its total quotient module $q(M)$ is a vn-regular $R_{S}$ module, where $S=R-Z(M)$. Moreover, $M$ is said to be a wq-regular module if for each $m \in M$, there is an $a \in R$ such that

$$
\operatorname{ann}_{M}\left(a n n_{R}(m)\right)=a n n_{M}(a) .
$$

A submodule $N$ of $M$ is said to be a $*$-submodule if

$$
N=O(S)=\{m \in M: s m=0 \text { for some } s \in S\}
$$

for some m.c.s $S \subseteq R$. $N$ is said to be an $\alpha$-submodule if for each $m_{1}, m_{2} \in N$ with $\operatorname{ann}_{R}\left(m_{1}\right) \cap \operatorname{ann}_{R}\left(m_{2}\right)=\operatorname{ann}_{R}\left(m_{3}\right)$, we have $m_{3} \in N$. Also $N$ is called an annihilator submodule if $\operatorname{ann}_{M}\left(\operatorname{ann}_{R}(N)\right)=N$. We study relations between these submodules and establish many characterizations of wq-regular modules in terms of $*$-submodules, $\alpha$-submodules and annihilator submodules (see Theorem 2.9-2.31). Also we prove that if $q(M)$ is a finitely generated multiplication module (not necessarily $M$ is) and $M$ is a non-torsion module, then $M$ is a quasi regular module if and only if $M$ is a reduced wq-regular module (compare the result [4, Theorem 2.2]). We also investigate whether the notion of wq-regular modules is invariant under homomorphism and direct products. In Section 3, we determine when the trivial extension $R \propto M$ (idealization) of $M$ is quasi regular and wq-regular, respectively (see Proposition 3.1 and Theoerem 3.4). In Section 4 , we investigate the extension of wq-regular modules. In particular, we show that when polynomial modules and formal power series modules are wq-regular (see Theorem 4.6). 


\section{Characterizations of quasi regular modules}

Throughout the section, we will examine $*$-submodules, $\alpha$-submodules, annihilator submodules and use them to characterize wq-regular modules.

Definition 2.1. Let $q(M)$ be the total quotient module of an $R$-module $M$. Then

(i) $M$ is called a quasi regular module if its total quotient module is vn-regular.

(ii) $M$ is called a wq-regular module if for each $m \in M$, there is an $a \in R$ such that $\operatorname{ann}_{M}\left(\operatorname{ann}_{R}(m)\right)=\operatorname{ann}_{M}(a)$.

Example 2.2. (i) Every torsion free module is wq-regular. To see this, take a nonzero element $m \in M$. Then $\operatorname{ann}_{R}(m)=0$, and so $\operatorname{ann}_{M}\left(a n n_{R}(m)\right)=M=\operatorname{ann}_{M}(0)$.

(ii) Every simple module is a wq-regular module. Assume $M$ is a simple $R$-module. Then $R m=M$ or $R m=0$ for every $m \in M$. If $R m=0$, then $\operatorname{ann}_{M}\left(\operatorname{ann}_{R}(m)\right)=0=$ $a n n_{M}(1)$. Otherwise, we would have $\operatorname{ann}_{M}\left(a n n_{R}(m)\right)=M=a n n_{M}(0)$.

(iii) Assume $R$ is a principal ideal ring. Then for any $m \in M$, $a n n_{R}(m)=(a)$ for some $a \in R$. Then we can conclude that $a n n_{M}\left(a n n_{R}(m)\right)=a n n_{M}(a)$. Hence every module over a principal ideal ring $R$ is wq-regular.

Example 2.3. (i) Every vn-regular module is a quasi-regular module. To see this, take a vn-regular $R$-module $M$. Let $\frac{m}{s} \in q(M)$ for some $m \in M, s \in S=R-Z(M)$. Then note that $R_{S}\left(\frac{m}{s}\right)=(R m)_{S}$. Also we have $R m=x M=x^{2} M$ for some $x \in R$ because $M$ is vn-regular. Then we can conclude that

$$
\begin{aligned}
R_{S}\left(\frac{m}{s}\right) & =(R m)_{S}=(x M)_{S}=\frac{x}{1} q(M) \\
& =\left(x^{2} M\right)_{S}=\left(\frac{x}{1}\right)^{2} q(M) .
\end{aligned}
$$

Hence, $M$ is quasi regular $R$-module.

(ii) Every simple module is vn-regular [8, Example 2], hence a quasi regular module by (i). In particular, the $\mathbb{Z}$-module $\mathbb{Z}_{p}$ is a quasi regular module for each prime number $p$.

(iii) Let $n>1$ be a square free integer, i.e, $n=p_{1} p_{2} \cdots p_{r}$, where $p_{i}$ 's are distinct prime numbers. Consider the $\mathbb{Z}$-module $\mathbb{Z}_{n}$. Then by [8, Example 5$], \mathbb{Z}_{n}$ is vn-regular and thus a quasi regular module by (i).

(iv) Let $n>1$ be a non-square free integer. We may assume that $n=p_{1}^{\alpha_{1}} p_{2}^{\alpha_{2}} \ldots p_{r}^{\alpha_{r}}$ for some distinct prime numbers $p_{1}, p_{2}, \ldots, p_{r}$, where $\alpha_{1} \geq 2$ and $\alpha_{2}, \alpha_{3}, \ldots, \alpha_{r} \geq 1$. Consider the $\mathbb{Z}$-module $\mathbb{Z}_{n}$. Then note that $Z\left(\mathbb{Z}_{n}\right)=p_{1} \mathbb{Z} \cup p_{2} \mathbb{Z} \cup \cdots \cup p_{r} \mathbb{Z}$ is a union of prime ideals of $\mathbb{Z}$. Now, take $S=\mathbb{Z}-Z\left(\mathbb{Z}_{n}\right)$. Then it is clear that $q\left(\mathbb{Z}_{n}\right)$ is a finitely generated multiplication $\mathbb{Z}_{S}$-module. Since $\mathbb{Z}_{n}$ is not a reduced ring, by [4, Theorem 2.2] its total quotient ring is not vn-regular. Now, it can be easily verified that

$$
\bar{S}=\pi(S)=\left\{a+n \mathbb{Z}: \operatorname{gcd}\left(a, p_{i}\right)=1 \text { for each } 1 \leq i \leq r\right\}
$$

is the set of regular elements of $\mathbb{Z} / n \mathbb{Z}$, where $\pi: \mathbb{Z} \rightarrow \mathbb{Z} / n \mathbb{Z}$ is the canonical homomorphism. Furthermore,

$$
a n n_{\mathbb{Z}_{S}}\left(q\left(\mathbb{Z}_{n}\right)\right)=\left(a n n_{\mathbb{Z}}\left(\mathbb{Z}_{n}\right)\right)_{S}=(n \mathbb{Z})_{S}
$$

and also $\mathbb{Z}_{S} / \operatorname{ann}_{\mathbb{Z}_{S}}\left(q\left(\mathbb{Z}_{n}\right)\right) \cong(\mathbb{Z} / n \mathbb{Z})_{\bar{S}}$. Again by $\left[4\right.$, Theorem 2.2], $\mathbb{Z}_{S} / \operatorname{ann}_{\mathbb{Z}_{S}}\left(q\left(\mathbb{Z}_{n}\right)\right)$ is not a vn-regular ring. Then by [8, Theorem 1$], q\left(\mathbb{Z}_{n}\right)$ is not a vn-regular $\mathbb{Z}_{S}$-module. Hence, $\mathbb{Z}_{n}$ is not a quasi regular $\mathbb{Z}$-module but wq-regular.

Definition 2.4. Let $N$ be a submodule of an $R$-module $M$. Then,

(i) $N$ is called a $*$-submodule if $N=O(S)=\{m \in M: s m=0$ for some $s \in S\}$, where $S \subseteq R$ is a m.c.s of $R$.

(ii) $\left(N:_{R} M\right)$ is a $*$-ideal if it is a $*$-submodule of the $R$-module $R$.

Let $N$ be a submodule of $M$. Then $N$ is called an $m$-submodule if $N=\left(N:_{R} M\right) M$. Note that an $R$-module $M$ is called a multiplication module if each submodule is an $m$-submodule [3]. 
Lemma 2.5. (i) Let $M$ be a non-torsion module and $N$ a *-submodule of $M$. Then $(N: M)$ is a $*$-ideal of $R$.

(ii) Let $N$ be a prime $m$-submodule of $M$ in which $(N: M)$ is a $*$-ideal. Then $N$ is a *-submodule.

Proof. (i) Assume $N$ is a $*$-submodule of $M$. Then $N=O(S)$ for some m.c.s $S$ of $R$. As $M$ is non-torsion, we get $a n n_{R}(m)=0$ for some $m \in M$. Let $r \in\left(N:_{R} M\right)$. Then $r m \in N$, and so $s(r m)=0$ for some $s \in S$. As $\operatorname{ann}_{R}(m)=0$, we have $s r=0$. Now set $\overleftarrow{O(S)}=\{x \in R: s x=0$ for some $s \in S\}$. Note that $r \in \overleftarrow{O(S)}$, and so $\left(N:_{R} M\right) \subseteq$ $\overleftarrow{O(S)}$. Let $x \in \overleftarrow{O(S)}$. Then $s x=0$ for some $s \in S$. This implies that $s(x M)=0$, and so $x M \subseteq O(S)=N$ and this yields $x \in\left(N:_{R} M\right)$. Accordingly, $\left(N:_{R} M\right)=\overleftarrow{O(S)}$ is a *-ideal of $R$.

(ii) Since $(N: M)$ is a $*$-ideal, $\left(N:_{R} M\right)=\overleftarrow{O(S)}=\{x \in R: s x=0$ for some $s \in S\}$, where $S$ is a m.c.s of $R$. Now, we will show that $N=O(S)$. Let $m \in N$. Since $N=\left(N:_{R} M\right) M$, we get $m=\sum_{i=1}^{n} a_{i} m_{i}, a_{i} \in\left(N:_{R} M\right)$ and $m_{i} \in M$. As $\left(N:_{R} M\right)=$ $\overleftarrow{O(S)}$, there is $s_{i} \in S$ such that $s_{i} a_{i}=0$ for each $i=1,2, \ldots, n$. Put $s=s_{1} s_{2} \ldots s_{n}$. Then note that $s m=\sum_{i=1}^{n}\left(s a_{i}\right) m_{i}=0$, and so $m \in O(S)$. Then we conclude that $N \subseteq O(S)$. For the converse, take $m \in O(S)$. Then $s m=0$ for some $s \in S$. It is clear that $S \cap\left(N:_{R}\right.$ $M)=\emptyset$ since $\left(N:_{R} M\right)=\overleftarrow{O(S)}$ and $0 \notin S$. This implies $s \notin\left(N:_{R} M\right)$, and so $m \in N$ as $N$ is a prime submodule. Accordingly, $N=O(S)$.

A submodule $N$ of an $R$-module $M$ is said to be a Baer submodule if for each $m \in$ $N, \operatorname{ann}_{M}\left(a n n_{R}(m)\right) \subseteq N$.

Definition 2.6. A submodule $N$ of an $R$-module $M$ is said to be an $\alpha$-submodule if for each $m_{1}, m_{2} \in N$ with $a n n_{R}\left(m_{1}\right) \cap a n n_{R}\left(m_{2}\right)=a n n_{R}\left(m_{3}\right)$, we have $m_{3} \in N$.

Baer ideals and $\alpha$-ideals are defined as Baer submodules and $\alpha$-submodules of the $R$ module $R$, respectively. In fact, $\alpha$-ideals are exactly strong Baer ideals of $R$ [7].

Proposition 2.7. (i) Every *-submodule is a Baer submodule.

(ii) Assume $M$ is a module over a reduced ring $R$ satisfying the condition: for each $m \in$ $M$, $a n n_{R}(m)=a n n_{R}(r)$ for some $r \in R$. Then every $\alpha$-submodule is a Baer submodule.

(iii) Every $*$-submodule is an $\alpha$-submodule.

Proof. (i) Assume that $N=O(S)$ for some m.c.s $S$ of $R$. Take $m \in N$. Then there is an $s \in S$ so that $s m=0$. Let $m^{\prime} \in \operatorname{ann}_{M}\left(a n n_{R}(m)\right)$. Then we have $\operatorname{ann}_{R}(m) m^{\prime}=0$, and so $s m^{\prime}=0$ since $s \in a n n_{R}(m)$. This implies that $m^{\prime} \in O(S)=N$. Thus $N$ is a Baer submodule.

(ii) Let $m^{\prime} \in a n n_{M}\left(a n n_{R}(m)\right)$ with $m \in N$. Then $a n n_{R}(m) m^{\prime}=0$, and so $a n n_{R}(m) \subseteq$ $\operatorname{ann}_{R}\left(m^{\prime}\right)$. By assumption, we have $a n n_{R}(m)=a n n_{R}(x)$ and $a n n_{R}\left(m^{\prime}\right)=a n n_{R}(y)$ for some $x, y \in R$. Then $\operatorname{ann}_{R}(x) \subseteq \operatorname{ann}_{R}(y)$. Since $R$ is a reduced ring, we have $a n n_{R}\left(m^{\prime}\right)=$ $\operatorname{ann}_{R}(y)=\operatorname{ann}_{R}(x y)=a n n_{R}(y m)$. Since $N$ is an $\alpha$-submodule and $y m \in N$, we get $m^{\prime} \in N$, and so $a n n_{M}\left(a n n_{R}(m)\right) \subseteq N$. Accordingly, $N$ is a Baer submodule.

(iii) Let $N$ be a $*$-submodule, i.e, $N=O(S)$ for some m.c.s $S$ of $R$. Assume that $\operatorname{ann}_{R}(m) \cap \operatorname{ann}_{R}\left(m^{\prime}\right)=\operatorname{ann}_{R}\left(m^{\prime \prime}\right)$ with $m, m^{\prime} \in N$ and $m^{\prime \prime} \in M$. Then there are $s, s^{\prime} \in$ $S$ such that $s m=s^{\prime} m^{\prime}=0$. Now put $t=s s^{\prime}$. Then $t \in S$ and $t \in \operatorname{ann}_{R}(m) \cap a n n_{R}\left(m^{\prime}\right)$ and this yields that $t m^{\prime \prime}=0$. Thus we have $m^{\prime \prime} \in O(S)=N$. Accordingly, $N$ is an $\alpha$-submodule of $M$.

Remember that $M$ is said to be a reduced $R$-module if for $r \in R, m \in M$ and $r m=0$, we have $r M \cap R m=0$, or equivalently, $r^{2} m=0$ implies $r m=0$ [9]. 
Proposition 2.8. (i) Let $N$ be a prime $m$-submodule of a non-torsion module $M$. Then $N$ is a *-submodule if and only if $\left(N:_{R} M\right)$ is a *-ideal of $R$.

(ii) Let $M$ be a non-torsion reduced module over a quasi-regular ring $R$. Then any prime $m$-submodule $N$ of $M$ is a Baer submodule if and only if $\left(N:_{R} M\right)$ is a Baer ideal.

Proof. (i) It can be obtained from Lemma 2.5 (i) and (ii).

(ii) Assume $\left(N:_{R} M\right)$ is a Baer ideal and $N$ is a prime $m$-submodule of $M$. First note that $R$ is a reduced ring. By [7, Corollary 3], $\left(N:_{R} M\right)$ is a $*$-ideal of $R$. By Lemma 2.5 (ii), $N$ is a $*$-submodule. Then by Proposition 2.7 (i), $N$ is a Baer submodule of $M$. For the converse, assume $N$ is a Baer submodule. Let $r \in\left(N:_{R} M\right)$. As $M$ is non-torsion, we get $a n n_{R}(m)=0$ for some $m \in M$. Then note that $r m \in N$ and $a n n_{R}(r m)=a n n_{R}(r)$. As $N$ is a Baer submodule, we can conclude that $\operatorname{ann}_{M}\left(a n n_{R}(r m)\right)=a n n_{M}\left(a n n_{R}(r)\right) \subseteq N$. Now we will show that, for each ideal $I$ of $R,\left(\operatorname{ann}_{M}(I): M\right)=\operatorname{ann}_{R}(I)$. The containment $\operatorname{ann}_{R}(I) \subseteq\left(\operatorname{ann}_{M}(I): M\right)$ always holds. Let $x \in\left(\operatorname{ann}_{M}(I): M\right)$. Then $x M \subseteq \operatorname{ann}_{M}(I)$, and so $I(x M)=0$. This implies that $I(x m)=0$, and so $I x \subseteq a n n_{R}(m)=0$. Then we have $x \in \operatorname{ann}_{R}(I)$, which yields $\left(\operatorname{ann}_{M}(I): M\right)=\operatorname{ann}_{R}(I)$. Since $\operatorname{ann}_{M}\left(\operatorname{ann}_{R}(r)\right) \subseteq N$, we have $\left(a n n_{M}\left(a n n_{R}(r)\right):_{R} M\right)=\operatorname{ann}_{R}\left(a n n_{R}(r)\right) \subseteq\left(N:_{R} M\right)$. Thus $\left(N:_{R} M\right)$ is a Baer ideal.

We now characterize wq-regular modules in terms of $*$-submodules.

Theorem 2.9. Let $M$ be a reduced faithful module. Then $M$ is a wq-regular module if and only if $\operatorname{ann}_{M}\left(\operatorname{ann}_{R}(m)\right)$ is a *-submodule for each $m \in T(M)$.

Proof. Assume that $M$ is a wq-regular module. Take an element $m \in T(M)$. Then $a n n_{R}(m) \neq 0$. As $M$ is a wq-regular module, $a n n_{M}\left(a n n_{R}(m)\right)=a n n_{M}(r)$ for some $r \in R$. Since $M$ is faithful, $r \neq 0$. Otherwise, we would have $a n n_{R}(m)=a n n_{R}(M)=0$, a contradiction. As $M$ is a reduced module, $R$ is a reduced ring, and so $S=\left\{r^{n}: n \in\right.$ $\mathbb{N}\}$ is an m.c.s of $R$. Also note that $\operatorname{ann}_{M}\left(\operatorname{ann}_{R}(m)\right)=\operatorname{ann}_{M}(r)=O(S)$, and so $a n n_{M}\left(a n n_{R}(m)\right)$ is a $*$-submodule. For the converse, assume $a n n_{M}\left(a n n_{R}(m)\right)$ is a $*$ submodule for each $m \in T(M)$. Let $m \in M$. If $a n n_{R}(m)=0$, then $a n n_{M}\left(a n n_{R}(m)\right)=$ $M=\operatorname{ann}_{M}(0)$. Assume that $m \in T(M)$. By assumption, $\operatorname{ann}_{M}\left(a n n_{R}(m)\right)=O(S)$ for some m.c.s $S$ of $R$. This yields $r m=0$ for some $r \in S$, which yields $a n n_{M}\left(a n n_{R}(m)\right) \subseteq$ $\operatorname{ann}_{M}(r)$. Let $m^{\prime} \in \operatorname{ann}_{M}(r)$. Then we have $r m^{\prime}=0$, and so $m^{\prime} \in O(S)=\operatorname{ann}_{M}\left(a n n_{R}(m)\right)$. Thus $\operatorname{ann}_{M}\left(\operatorname{ann}_{R}(m)\right)=\operatorname{ann}_{M}(r)$.

Proposition 2.10. Let $M$ be a non-torsion wq-regular module. Then $R$ is a wq-regular ring and for each $m \in M$, there is an $r \in R$ such that $\operatorname{ann}_{R}(m)=\operatorname{ann}_{R}(r)$.

Proof. Let $r \in R$. Since $M \neq T(M)$, we get $a n n_{R}(m)=0$ for some $m \in M$ and also note that $a n n_{R}(r)=a n n_{R}(r m)$. As $M$ is wq-regular, there is an $s \in R$ such that $a n n_{M}(s)=\operatorname{ann}_{M}\left(a n n_{R}(r m)\right)$, and so $a n n_{M}(s)=a n n_{M}\left(a n n_{R}(r)\right)$. Then we conclude that

$$
\begin{aligned}
\operatorname{ann}_{R}\left(\operatorname{ann}_{R}(r)\right) & =\left(\operatorname{ann}_{M}\left(\operatorname{ann} n_{R}(r)\right):_{R} M\right) \\
& =\left(\operatorname{ann}_{M}(s):_{R} M\right) \\
& =\operatorname{ann}_{R}(s) .
\end{aligned}
$$

Therefore, $R$ is a wq-regular ring. Take an element $m^{*} \in M$. As $M$ is wq-regular, $\operatorname{ann}_{M}\left(a n n_{R}\left(m^{*}\right)\right)=a n n_{M}(a)$ for some $a \in R$. This yields $a n n_{R}\left(a n n_{R}\left(m^{*}\right)\right)=a n n_{R}(a)$, and so $a n n_{R}\left(m^{*}\right)=a n n_{R}\left(a n n_{R}(a)\right)=a n n_{R}(b)$ for some $b \in R$ because $R$ is a wq-regular ring.

Proposition 2.11. Assume $M$ is a non-torsion module and ann $n_{M}(I)$ is an m-submodule of $M$ for each ideal $I$ of $R$. If $R$ is a wq-regular ring and for each $m \in M$, ann $n_{R}(m)=$ ann $_{R}(r)$ for some $r \in R$, then $M$ is a wq-regular module. 
Proof. Assume $R$ is a wq-regular ring and for each $m \in M$, $a n n_{R}(m)=a n n_{R}(r)$ for some $r \in R$. Let $m \in M$. Then by assumption, $a n n_{R}(m)=a n n_{R}(r)$ for some $r \in R$. As $R$ is wqregular, there is an $s \in R$ so that $a n n_{R}\left(a n n_{R}(r)\right)=\operatorname{ann}_{R}(s)$, and so $\left(a n n_{M}\left(a n n_{R}(r)\right):_{R}\right.$ $M)=\operatorname{ann}_{R}(s)$. This yields that $\left(\operatorname{ann}_{M}\left(a n n_{R}(r)\right):_{R} M\right)=\left(\operatorname{ann}_{M}(s):_{R} M\right)$. Since $\operatorname{ann}_{M}(I)$ is an $m$-submodule for each ideal $I$ of $R$, we get

$$
\begin{aligned}
\operatorname{ann}_{M}\left(\operatorname{ann}_{R}(r)\right) & =\left(\operatorname{ann}_{M}\left(\operatorname{ann}_{R}(r)\right):_{R} M\right) M \\
& =\left(\operatorname{ann}_{M}(s):_{R} M\right) M \\
& =\operatorname{ann}_{M}(s) .
\end{aligned}
$$

Accordingly, $M$ is a wq-regular module.

The following example shows that an $R$-module satisfying all conditions in Proposition 2.11 may not be a multiplication module.

Example 2.12. Consider a torsion free module but not a multiplication module, e.g, a vector space $V$ over a field $F$ with $\operatorname{dim}_{F}(V)>1$. Note that $V$ is a non-torsion module and for each $0 \neq m \in V, \operatorname{ann}_{F}(m)=0=a n n_{F}(1)$. Also it is easily seen that $a n n_{V}(0)=V$ and $\operatorname{ann}_{V}(F)=0$ are m-submodules of $V$. But $V$ can not be a multiplication module.

The next Theorem 2.13 characterizes wq-regular modules in terms of wq-regular rings.

Theorem 2.13. Let $M$ be a non-torsion module and $a n_{M}(I)$ is an $m$-submodule for each ideal I of $R$. Then the followings are equivalent:

(i) $M$ is wq-regular module.

(ii) $R$ is wq-regular ring and for each $m \in M$, there is an $r \in R$ such that ann $n_{R}(m)=$ $\operatorname{ann}_{R}(r)$.

Proof. It can be obtained from Proposition 2.10 and Proposition 2.11.

Definition 2.14. Let $M$ be a finitely generated $R$-module. Then,

(i) $M$ is said to satisfy the condition (\#) if $K$ is a minimal prime submodule, then $K=\left(K:_{R} M\right) M$.

(ii) $M$ is said to satisfy the condition $(P)$ if $\bigcap(P M)=(\bigcap P) M$ for all prime ideals $P$ minimal over $a n n_{R}(M)$.

(iii) $M$ is said to satisfy the condition (\#\#) if it satisfies the condition (\#) and (P).

Remark that a finitely generated multiplication module satisfies the conditions (\#) and (\#\#). But the converse is not true.

Example 2.15. Every finite dimensional vector space satisfies (\#) and (\#\#). In particular, consider the Euclidean Plane $\mathbb{R}$-module $\mathbb{R}^{2}$. Since 0 is a prime submodule, it is a minimal prime submodule. It is straightforward that the $\mathbb{R}$-module $\mathbb{R}^{2}$ satisfies (\#) and $(\# \#)$. But it is not a multiplication module.

Proposition 2.16. Let $M$ be a finitely generated module and $K$ be a submodule of $M$. Assume that $M$ satisfies the condition (\#). Then

(i) If $P$ is a prime minimal over ann $n_{R}(M)$, then $P M$ is a minimal prime submodule.

(ii) If $K$ is a minimal prime submodule, then $\left(K:_{R} M\right)$ is a prime ideal minimal over $\operatorname{ann}_{R}(M)$.

Proof. (i) Assume $P$ is a prime ideal minimal over $a n_{R}(M)$. By [11, Proposition 8], $\left(P M:_{R} M\right)=P$. By [12, Theorem 3.3], $P M$ is contained in some prime submodule $N$ with $\left(N:_{R} M\right)=P$. Again by Zorn's Lemma, $P M$ is contained in $N_{1}$ where $N_{1}$ is a prime submodule minimal over $P M$ such that $\left(N_{1}:_{R} M\right)=P$. The reader can easily verify that $N_{1}$ is a minimal prime submodule.

(ii) Assume $K$ is a minimal prime submodule. Thus $\left(K:_{R} M\right)$ is a prime ideal. Since $\operatorname{ann}_{R}(M)$ is contained in $\left(K:_{R} M\right)$, there is a prime $P$ minimal over $a n n_{R}(M)$ such that 
$P$ is contained in $\left(K:_{R} M\right)$. So $P M$ is contained in $K$. By (i), $P M$ is a minimal prime submodule, thereby $P M=K$. Again $\left(K:_{R} M\right)=\left(P M:_{R} M\right)=P$ by [11, Proposition 8].

Proposition 2.17. Let $M$ be a finitely generated module and $I$ be an ideal containing ann $_{R}(M)$. Assume that every prime submodule minimal over IM is an m-submodule. Then

(i) If $P$ is minimal over $I$, then $P M$ is a prime minimal over $I M$.

(ii) If $K$ is minimal over $I M$, then $\left(K:_{R} M\right)$ is minimal over $I$.

Proof. The proof is similar to the proof of Proposition 2.16.

We shall now prove several lemmas that we need.

Lemma 2.18. Let $M$ be a non-torsion wq-regular module over a reduced ring $R$. Then $M$ satisfies annihilator condition, i.e, for any $m_{1}, m_{2} \in M$, there is an $m_{3} \in M$ such that

$$
\operatorname{ann}_{R}\left(m_{1}\right) \cap \operatorname{ann}_{R}\left(m_{2}\right)=\operatorname{ann}_{R}\left(m_{3}\right) .
$$

Proof. By Proposition 2.10, $a n n_{R}\left(m_{1}\right)=a n n_{R}\left(r_{1}\right)$ and $a n n_{R}\left(m_{2}\right)=a n n_{R}\left(r_{2}\right)$ for some $r_{1}, r_{2} \in R$. Since $R$ is a reduced wq-regular ring, it is quasi regular, and so satisfies annihilator condition, i.e, $\operatorname{ann}_{R}\left(r_{1}\right) \cap a n n_{R}\left(r_{2}\right)=a n n_{R}\left(r_{3}\right)$ for some $r_{3} \in R$. Choose $m \in M-T(M)$. Then ann $_{R}\left(r_{3}\right)=\operatorname{ann}_{R}\left(r_{3} m\right)$. Put $m_{3}=r_{3} m$. So we have ann $_{R}\left(m_{1}\right) \cap$ $\operatorname{ann}_{R}\left(m_{2}\right)=a n n_{R}\left(m_{3}\right)$. Thus $M$ satisfies annihilator condition.

Lemma 2.19. Let $N$ be a Baer submodule of an $R$-module $M$. If ann $n_{R}(m)=$ ann $_{R}(r)$ with $m \in N$, then $r \in(N: R M)$.

Proof. Since $N$ is a Baer submodule, we have $\operatorname{ann}_{M}\left(a n n_{R}(m)\right)=\operatorname{ann}_{M}\left(a n n_{R}(r)\right) \subseteq N$, and so $\left(\operatorname{ann}_{M}\left(\operatorname{ann}_{R}(r)\right):_{R} M\right) \subseteq\left(N:_{R} M\right)$. This yields $r \in\left(N:_{R} M\right)$.

Lemma 2.20. Assume that $M$ is a finitely generated module satisfying the condition $(P)$ and $I$ is an ideal containing $\operatorname{ann}_{R}(M)$. Assume that every prime submodule minimal over $I M$ is an $m$-submodule. Then $\operatorname{rad}(I M)=\operatorname{rad}(I) M$.

Proof. $\operatorname{rad}(I M)=\bigcap_{N_{\alpha} \in \operatorname{Min}(I M)} N_{\alpha}=\left[\bigcap\left(N_{\alpha}:_{R} M\right) M\right]=\left[\cap\left(N_{\alpha}:_{R} M\right)\right] M=\sqrt{I} M$.

Definition 2.21. An $m$-submodule $N$ is said to be a strong $m$-submodule if all prime submodules minimal over $N$ are $m$-submodules.

Note that $M$ is a multiplication module if and only if every submodule is a strong $m$-submodule.

Lemma 2.22. Assume that $M$ is a finitely generated reduced module and $N$ is a strong m-submodule which is also a Baer submodule. Then every prime submodule minimal over $N$ is a Baer submodule.

Proof. Let $N^{\prime}$ be a minimal over $N$. Assume $a n n_{R}(m) \subseteq a n n_{R}\left(m^{\prime}\right)$ with $m \in N^{\prime}$. By Proposition 2.17, $\left(N^{\prime}:_{R} M\right)$ is a minimal over $\left(N:_{R} M\right)$. As $m \in N^{\prime}=\left(N^{\prime}:_{R} M\right) M, m=$ $\sum_{i=1}^{n} a_{i} m_{i}$ for some $a_{i} \in\left(N^{\prime}:_{R} M\right)$ and $m_{i} \in M$. Then there exist $b_{i} \notin\left(N^{\prime}:_{R} M\right)$ and $n_{i} \in \mathbb{N}$ so that $a_{i}^{n_{i}} b_{i} \in\left(N:_{R} M\right)$. Since $N$ is a Baer submodule, $\left(N:_{R} M\right)=\sqrt{\left(N:_{R} M\right)}$, and so $a_{i} b_{i} \in\left(N:_{R} M\right)$. Put $b=b_{1} b_{2} \ldots b_{n}$. Then $b \notin\left(N^{\prime}:_{R} M\right)$ and $a_{i} b \in\left(N:_{R} M\right)$, and so $a_{i} b m_{i} \in\left(N:_{R} M\right) M=N$, and we have $b m \in N$. Since $a n n_{R}(b m) \subseteq a n n_{R}\left(b m^{\prime}\right)$ and $N$ is a Baer submodule, $b m^{\prime} \in N \subseteq N^{\prime}$. As $b \notin\left(N^{\prime}: R M\right)$, we deduce $m^{\prime} \in N^{\prime}$, and so $N^{\prime}$ is a Baer submodule.

Lemma 2.23. Let $M$ be a finitely generated reduced module satisfying the condition (P) and $N$ a strong $m$-submodule which is also a Baer submodule. Then $N$ is the intersection of prime Baer submodules. 
Proof. It can be obtained from Lemma 2.20 and Lemma 2.22.

Lemma 2.24. Assume $M$ is a non-torsion reduced module and $N$ is a Baer submodule which is also a prime submodule. Then $\left(N:_{R} M\right)$ is a prime and Baer ideal.

Proof. We claim that $R$ is a reduced ring. Assume that $a^{2}=0$ for some $a \in R$. As $M$ is a non-torsion module, we have $\operatorname{ann}_{R}(m)=0$ for some $m \in M$. Then $a^{2} m=0$ and thereby $a m=0$ since $M$ is reduced. This yields $a=0$, and thus $R$ is a reduced ring. Let $a n n_{R}(x)=a n n_{R}(y)$ for some $x \in\left(N:_{R} M\right)$ and $y \in R$. Then $a n n_{R}(x m)=a n n_{R}(x)=$ $a n n_{R}(y m)$. Since $x m \in N$ and $N$ is a Baer submodule, we conclude that $y m \in N$. Also note that $m \notin N$. As $N$ is a prime submodule, $y \in\left(N:_{R} M\right)$. Then by [7, Lemma 1], $\left(N:_{R} M\right)$ is a Baer ideal. Since $N$ is a prime submodule, it follows that $\left(N:_{R} M\right)$ is a Baer and prime ideal.

Lemma 2.25. Let $M$ be a finitely generated reduced non-torsion wq-regular module. Further, assume $M$ satisfies the condition $(P)$. Let $N$ be a strong $m$-submodule which is also a Baer submodule. Then $N$ is an $\alpha$-submodule.

Proof. Assume $\operatorname{ann}_{R}\left(m_{1}\right) \cap a n n_{R}\left(m_{2}\right)=a n n_{R}\left(m_{3}\right)$ with $m_{1}, m_{2} \in N$ but $m_{3} \notin N$. By Lemma 2.23, there is a prime Baer submodule $N^{\prime}$ with $m_{3} \notin N^{\prime}$. By Proposition 2.10, $\operatorname{ann}_{R}\left(m_{i}\right)=\operatorname{ann}_{R}\left(r_{i}\right)$ for some $r_{i} \in R, i=1,2,3$. By Lemma 2.19, $r_{1}, r_{2} \in\left(N^{\prime}:_{R}\right.$ $M)$. Since $R$ is quasi-regular, there are $r_{1}^{\prime}, r_{2}^{\prime} \in R$ so that $r_{1} r_{1}^{\prime}=0=r_{2} r_{2}^{\prime}$ with ann ${ }_{R}\left(r_{1}+\right.$ $\left.r_{1}^{\prime}\right)=\operatorname{ann}_{R}\left(r_{2}+r_{2}^{\prime}\right)=0$. Since $r_{1}^{\prime} r_{2}^{\prime} m_{3}=0 \in N^{\prime}$ and $m_{3} \notin N^{\prime}$, we have either $r_{1}^{\prime} \in\left(N^{\prime}:_{R}\right.$ $M)$ or $r_{2}^{\prime} \in\left(N^{\prime}:_{R} M\right)$. By Lemma 2.24, $\left(N^{\prime}:_{R} M\right)$ is a Baer ideal and either $r_{1}+r_{1}^{\prime} \in$ $\left(N^{\prime}:_{R} M\right)$ or $r_{2}+r_{2}^{\prime} \in\left(N^{\prime}:_{R} M\right)$, a contradiction. Thus $N$ is an $\alpha$-submodule.

Lemma 2.26. Let $M$ be a non-torsion wq-regular module over a reduced ring $R$. Then every $\alpha$-submodule is a *-submodule.

Proof. Let $N$ be an $\alpha$-submodule. Put $S=\left\{r \in R: a n n_{R}(m)=a n_{R}\left(a n n_{R}(r)\right)\right.$ for some $m \in N\}$. Note that by Proposition 2.10, for each $m \in M$, $a n n_{R}(m)=a n n_{R}(r)$ for some $r \in R$. Also by Proposition 2.7, $N$ is a Baer submodule. It can be easily seen that $S$ is a m.c.s. Let $m \in N$. Then $a n n_{R}(m)=a n n_{R}(a)$ for some $a \in R$. As $R$ is a wq-regular, $a n n_{R}(a)=a n n_{R}\left(a n n_{R}(r)\right)$ for some $r \in R$. So $a n n_{R}(m)=a n n_{R}\left(a n n_{R}(r)\right)$ and this implies that $r m=0$ and $r \in S$. Then we have $m \in O(S)$, i.e, $N \subseteq O(S)$. Let $m^{\prime} \in O(S)$. Then we have $r^{\prime} m^{\prime}=0$ for some $r^{\prime} \in S$. Also $a n n_{R}(m)=a n n_{R}\left(a n n_{R}\left(r^{\prime}\right)\right)$ for some $m \in N$. As $R$ is wq-regular, $a n n_{R}\left(m^{\prime}\right)=a n n_{R}\left(a^{\prime}\right)$ for some $a^{\prime} \in R$. Then $r^{\prime} \in$ $\operatorname{ann}_{R}\left(a^{\prime}\right)$, and so $a n n_{R}\left(a n n_{R}\left(a^{\prime}\right)\right)=\operatorname{ann}_{R}\left(a n n_{R}\left(m^{\prime}\right)\right) \subseteq a n n_{R}\left(r^{\prime}\right)=\operatorname{ann}_{R}\left(a n n_{R}(m)\right)$. Since $m \in N$ and $N$ is a Baer submodule, we have $m^{\prime} \in N$ and thus $N=O(S)$. Hence $N$ is a $*$-submodule.

Lemma 2.27. Let $M$ be an R-module. Assume that every $\alpha$-submodule is also a *submodule. Then $M$ is a wq-regular.

Proof. First we prove that, $N=\operatorname{ann}_{M}\left(a n n_{R}(m)\right)$ is an $\alpha$-submodule for each $m \in$ $T(M)$. Let $a n n_{R}\left(m^{\prime}\right) \cap a n n_{R}\left(m^{\prime \prime}\right)=a n n_{R}\left(m^{\prime \prime \prime}\right)$ with $m^{\prime}, m^{\prime \prime} \in N$. Then we have $a n n_{R}(m) \subseteq$ $\operatorname{ann}_{R}\left(m^{\prime}\right)$ and $a n n_{R}(m) \subseteq \operatorname{ann}_{R}\left(m^{\prime \prime}\right)$. This implies that $a n n_{R}(m) \subseteq a n n_{R}\left(m^{\prime}\right) \cap a n n_{R}\left(m^{\prime \prime}\right)=$ $a n n_{R}\left(m^{\prime \prime \prime}\right)$ and this yields that $m^{\prime \prime \prime} \in a n n_{M}\left(a n n_{R}\left(m^{\prime \prime \prime}\right)\right) \subseteq a n n_{M}\left(a n n_{R}(m)\right)=N$. Thus $N$ is an $\alpha$-submodule. The rest is similar to Theorem 2.9 .

The following Theorem 2.28 characterizes wq-regular modules in terms of $*$-submodules and $\alpha$-submodules.

Theorem 2.28. Let $M$ be a non-torsion reduced module. Then $M$ is a wq-regular module if and only if every Baer submodule is a $*$-submodule if and only if every $\alpha$-submodule is $a *$-submodule. 
Proof. It can be obtained from Lemma 2.26, Lemma 2.27, Proposition 2.7 and Theorem 2.9 .

Definition 2.29. Let $N$ be a submodule of $M$. Then $N$ is called an annihilator submodule if $\operatorname{ann}_{M}\left(a n n_{R}(N)\right)=N$. In particular, an annihilator ideal is an ideal $I$ of $R$ which is an annihilator submodule of the $R$-module $R$.

Note that a cyclic submodule $R m$ is an annihilator submodule if and only if it is a Baer submodule.

Lemma 2.30. Let $M$ be an R-module. Then,

(i) Every annihilator submodule is an $\alpha$-submodule.

(ii) Let $M$ be a non-torsion module and $N$ an annihilator submodule. Then $\left(N:_{R} M\right)$ is an annihilator ideal.

Proof. (i) Assume $N$ is an annihilator submodule, i.e, $N=a n n_{M}\left(a n n_{R}(N)\right)$. Suppose $\operatorname{ann}_{R}(m) \cap \operatorname{ann}_{R}\left(m^{\prime}\right)=a n n_{R}\left(m^{\prime \prime}\right)$ for some $m, m^{\prime} \in N$. This yields $a n n_{R}(N) m=$ $0=a n n_{R}(N) m^{\prime}$, and so $a n n_{R}(N) \subseteq a n n_{R}(m) \cap a n n_{R}\left(m^{\prime}\right)$. Then we can conclude that $\operatorname{ann}_{R}(N) \subseteq \operatorname{ann}_{R}\left(m^{\prime \prime}\right)$, and so $m^{\prime \prime} \in \operatorname{ann}_{M}\left(a n n_{R}\left(m^{\prime \prime}\right)\right) \subseteq a n n_{M}\left(a n n_{R}(N)\right)=N$. So that $N$ is an $\alpha$-submodule.

(ii) Let $N=a n n_{M}\left(a n n_{R}(N)\right)$. Since $M$ is non-torsion, $\left(N:_{R} M\right)=a n n_{R}\left(a n n_{R}(N)\right)$. Let $r \in \operatorname{ann}_{R}(N)$. Then $r N=0$, and so $r\left(N:_{R} M\right) M=0$. Choose $m \in M-T(M)$. This implies $r\left(N:_{R} M\right) m=0$, and so $r\left(N:_{R} M\right)=0$ and hence $r \in a n n_{R}\left(N:_{R} M\right)$. Then we can conclude $\operatorname{ann}_{R}\left(a n n_{R}\left(N:_{R} M\right)\right) \subseteq \operatorname{ann}_{R}\left(a n n_{R}(N)\right)$, and so $a n n_{R}\left(a n n_{R}\left(N:_{R}\right.\right.$ $M)) \subseteq\left(N:_{R} M\right)$. This implies that $\left(N:_{R} M\right)=a n n_{R}\left(a n n_{R}\left(N:_{R} M\right)\right)$. Consequently, $\left(N:_{R} M\right)$ is an annihilator ideal.

The following Theorem 2.31 characterizes wq-regular modules in terms of annihilator submodules.

Theorem 2.31. Let $M$ be a non-torsion module over a reduced ring $R$. Then $M$ is a wq-regular module if and only if every annihilator submodule is a $*$-submodule.

Proof. Assume $M$ is a wq-regular module. By Lemma 2.30, every annihilator submodule is an $\alpha$-submodule, and so by Lemma 2.26, every annihilator submodule is a $*$-submodule. For the converse, assume every annihilator submodule is a $*$-submodule. Let $m \in N$. Put $N=\operatorname{ann}_{M}\left(a n n_{R}(m)\right)$. Then it is easily seen that $N$ is an annihilator submodule and thus a $*$-submodule. Then there is a m.c.s $S$ of $R$ so that $a n n_{M}\left(a n n_{R}(m)\right)=O(S)$. The rest is similar to Theorem 2.9 .

We now study quasi regular modules.

Theorem 2.32. (i) Let $M$ be a non-torsion reduced wq-regular module. Assume that $q(M)$ is a multiplication module. Then $q(M)$ is a vn-regular module.

(ii) Assume that $q(M)$ is a finitely generated vn-regular module. Then $M$ is a reduced wq-regular module.

Proof. (i) Let $\frac{m}{t} \in q(M)$ and $S=R-Z(M)$. Put $N=R_{S}\left(\frac{m}{t}\right)$. As $q(M)$ is a multiplication module and $N$ is a finitely generated submodule of $q(M)$, then $N=J q(M)$ for some finitely generated ideal $J$ of $R_{S}$. Then there are $\frac{a_{1}}{s_{1}}, \ldots, \frac{a_{n}}{s_{n}} \in R_{S}$ such that $J=R_{S}\left(\frac{a_{1}}{s_{1}}\right)+\ldots+$ $R_{S}\left(\frac{a_{n}}{s_{n}}\right)$. Now, we will show that $R_{S}\left(\frac{a_{1}}{s_{1}}\right)=R_{S}\left(\frac{a_{1}}{s_{1}}\right)^{2}$, and so $R_{S}\left(\frac{a_{1}}{s_{1}}\right)=R_{S}\left(\frac{e_{1}}{t_{1}}\right)$ for some idempotent $\frac{e_{1}}{t_{1}} \in R_{S}$. As $M$ is non-torsion, we have $a n n\left(m^{*}\right)=0$ for some $m^{*} \in M$. Since $M$ is wq-regular, $\operatorname{ann}_{M}\left(\operatorname{ann}_{R}\left(a_{1} m^{*}\right)\right)=\operatorname{ann}_{M}\left(b_{1}\right)$ and thereby $\operatorname{ann}_{R}\left(\operatorname{ann}_{R}\left(a_{1} m^{*}\right)\right)=$ $\operatorname{ann}_{R}\left(b_{1}\right)$. Note that $a n_{R}\left(a_{1} m^{*}\right)=a n n_{R}\left(a_{1}\right)$, and so $a n_{R}\left(a n n_{R}\left(a_{1}\right)\right)=a n n_{R}\left(b_{1}\right)$. As $M$ is a reduced non-torsion module and $M$ is a wq-regular module, by Proposition 2.10 and [4, Theorem 2.1], $R$ is quasi-regular and thus $a_{1}+b_{1}$ is a regular element and $a_{1} x=$ $a_{1}^{2}$, where $x=a_{1}+b_{1}$. Now we will show that $x \in S$. Let $m^{\prime} \in M$ such that $x m^{\prime}=0$. Since 
$M$ is wq-regular, $M$ satisfies the condition $a n n_{R}\left(m^{\prime}\right)=a n n_{R}(r)$ for some $r \in R$, and so $x \in \operatorname{ann}_{R}\left(m^{\prime}\right)=\operatorname{ann}_{R}(r)$ and this yields that $x r=0$. Since $x$ is regular, $r=0$, and so $\operatorname{ann}_{R}(r)=R=\operatorname{ann}_{R}\left(m^{\prime}\right)$ and thus $m^{\prime}=0$ and this yields $x \in S$. This implies that $R_{S}\left(\frac{a_{1}}{s_{1}}\right)^{2}=R_{S}\left(\frac{a_{1}^{2}}{s_{1}^{2}}\right)=R_{S}\left(\frac{a_{1} x}{s_{1}^{2}}\right)=R_{S}\left(\frac{a_{1}}{s_{1}} \frac{x}{s_{1}}\right)=R_{S}\left(\frac{a_{1}}{s_{1}}\right)$ since $\frac{x}{s_{1}}$ is a unit element of $R_{S}$. Thus we have $R_{S}\left(\frac{a_{1}}{s_{1}}\right)=R_{S}\left(\frac{e_{1}}{t_{1}}\right)$ for some idempotent $\frac{e_{1}}{t_{1}} \in R_{S}$. Similarly, we get $R_{S}\left(\frac{a_{i}}{s_{i}}\right)=R_{S}\left(\frac{e_{i}}{t_{i}}\right)$ for some idempotent $\frac{e_{i}}{t_{i}} \in R_{S}$, and so $J=R_{S}\left(\frac{e}{s}\right)$ for some idempotent $\frac{e}{s} \in R_{S}$. Note that $\frac{e}{s}$ is weak idempotent $R_{S}$-module $q(M)$. Also $R_{S}\left(\frac{m}{t}\right)=J q(M)=$ $\frac{e}{s} q(M)$. Thus $q(M)$ is a vn-regular module.

(ii) By [8, Lemma 10], $q(M)$ is a reduced $R_{S}$-module, where $S=R-Z(M)$. Then it is easily seen that $M$ is reduced. Take an element $m \in M$. As $q(M)$ is a finitely generated vn-regular $R_{S}$-module, we deduce $R_{S}\left(\frac{m}{1}\right)=\frac{e}{s} q(M)$ for some weak idempotent $\frac{e}{s} \in R_{S}$. Note that $\left(1-\frac{e}{s}\right) \frac{e}{s} q(M)=\left(1-\frac{e}{s}\right) R_{S}\left(\frac{m}{1}\right)=0$, and so $\left(1-\frac{e}{s}\right) \frac{m}{1}=0$ and this yields $\left(1-\frac{e}{s}\right) \in \operatorname{ann}_{R_{S}}\left(\frac{m}{1}\right)$ and thus we have $\operatorname{ann}_{M_{S}}\left(\operatorname{ann}_{R_{S}}\left(\frac{m}{1}\right)\right) \subseteq \operatorname{ann}_{M_{S}}\left(1-\frac{e}{s}\right)$. Let $\frac{m^{*}}{s^{*}} \in \operatorname{ann}_{M_{S}}\left(1-\frac{e}{s}\right)$. Then we have $\frac{m^{*}}{s^{*}}=\frac{e}{s} \frac{m^{*}}{s^{*}}$. Take an element $\frac{r^{\prime}}{s^{\prime}} \in a n n_{R_{S}}\left(\frac{m}{1}\right)$. Then we conclude that $\frac{r^{\prime}}{s^{\prime}} \frac{e}{s} q(M)=0$. Note that $\frac{m^{*}}{s^{*}}=\frac{e}{s} \frac{m^{*}}{s^{*}} \in \frac{e}{s} q(M)$, and so $\frac{r^{\prime}}{s^{\prime}} \frac{m^{*}}{s^{*}}=0$ and this yields $\frac{m^{*}}{s^{*}} \in a n n_{M_{S}}\left(a n n_{R_{S}}\left(\frac{m}{1}\right)\right)$. Then we conclude that

$$
\begin{aligned}
\operatorname{ann}_{M_{S}}\left(\operatorname{ann}_{R_{S}}\left(\frac{m}{1}\right)\right) & =\left(\operatorname{ann}_{M}\left(\operatorname{ann}_{R}(m)\right)\right)_{S} \\
& =\operatorname{ann}_{M_{S}}\left(1-\frac{e}{s}\right) \\
& =\left(\operatorname{ann}_{M}(s-e)\right)_{S} .
\end{aligned}
$$

Then one can easily show that $a n n_{M}\left(a n n_{R}(m)\right)=a n n_{M}(s-e)$. Accordingly, $M$ is a wq-regular module.

Compare the following result with [4, Theorem 2.1].

Corollary 2.33. Let $M$ be a non-torsion module in which $q(M)$ is a finitely generated multiplication module. The followings are equivalent:

(i) $M$ is a quasi regular module.

(ii) $M$ is a reduced wq-regular module.

Proposition 2.34. Assume $f: M \rightarrow M^{\prime}$ is a monomorphism, where $M^{\prime}$ is a wq-regular module. Then $M$ is wq-regular.

Proof. Take $m \in M$. As $M^{\prime}$ is wq-regular, $a n n_{M^{\prime}}\left(a n n_{R}(f(m))=a n n_{M^{\prime}}(r)\right.$ for some $r \in$ $R$. Thus we have $r f(m)=f(r m)=0$, and so $r m=0$. This yields that $a n n_{M}\left(a n n_{R}(m)\right) \subseteq$ $\operatorname{ann}_{M}(r)$. Let $n \in \operatorname{ann}_{M}(r)$. Then we have $r n=0$, and so $r f(n)=f(r n)=0$, i.e, $f(n) \in \operatorname{ann}_{M^{\prime}}(r)=a n n_{M^{\prime}}\left(a n n_{R}(f(m))\right.$. Thus we conclude that $a n n_{R}(f(m)) f(n)=0$, and so $\operatorname{ann}_{R}(m) \subseteq \operatorname{ann}_{R}(n)$. This yields that $n \in \operatorname{ann}_{M}\left(a n n_{R}(n)\right) \subseteq \operatorname{ann}_{M}\left(a n n_{R}(m)\right)$. Accordingly, we have $\operatorname{ann}_{M}\left(a n n_{R}(m)\right)=\operatorname{ann}_{M}(r)$.

Corollary 2.35. Every submodule of a wq-regular module is wq-regular.

Proposition 2.36. Assume $M_{i}$ is an $R_{i}$-module for each $i \in \Delta$. Then $M=\prod_{i \in \Delta} M_{i}$ is a wq-regular $R=\prod_{i \in \Delta} R_{i}$-module if and only if $M_{i}$ is a wq-regular $R_{i}$-module for each $i \in \Delta$.

Proof. Assume that $M_{i}$ is a wq-regular $R_{i}$-module for each $i \in \Delta$. Let $\left(m_{j}\right)_{j \in \Delta} \in M$ and $\left(r_{j}\right)_{j \in \Delta} \in R$. For every $j \in \Delta$, ann $M_{j}\left(a n n_{R_{j}}\left(m_{j}\right)\right)=\operatorname{ann}_{M_{j}}\left(r_{j}\right)$ for some $r_{j} \in R_{j}$. Also note that

Thus we conclude that

$$
\operatorname{ann}_{M}\left(a n n_{R}\left(\left(m_{j}\right)_{j \in \Delta}\right)=\prod_{j \in \Delta} \operatorname{ann}_{M_{j}}\left(a n n_{R_{j}}\left(m_{j}\right)\right) .\right.
$$

$$
\operatorname{ann}_{M}\left(\operatorname{ann}_{R}\left(\left(m_{j}\right)_{j \in \Delta}\right)=\prod_{j \in \Delta} \operatorname{ann}_{M_{j}}\left(r_{j}\right)=\operatorname{ann}_{M}\left(\left(r_{j}\right)_{j \in \Delta}\right) .\right.
$$


Accordingly, $M$ is wq-regular. For the converse, assume $M$ is wq-regular. Let $m_{i} \in$ $M_{i}$. Put the sequence

Since $M$ is wq-regular, we have

$$
\left(n_{j}\right)_{j \in \Delta}= \begin{cases}m_{i} & ; j=i \\ 0 ; & j \neq i\end{cases}
$$

$$
\begin{aligned}
\operatorname{ann}_{M}\left(\operatorname{ann}_{R}\left(\left(n_{j}\right)_{j \in \Delta}\right)\right) & =\prod_{j \in \Delta} \operatorname{ann}_{M_{j}}\left(\operatorname{ann}_{R_{j}}\left(n_{j}\right)\right) \\
& =\operatorname{ann}_{M}\left(\left(r_{j}\right)_{j \in \Delta}\right) \\
& =\prod_{j \in \Delta} \operatorname{ann}_{M_{j}}\left(r_{j}\right)
\end{aligned}
$$

for some $\left(r_{j}\right)_{j \in \Delta} \in R$. This implies that $\operatorname{ann}_{M_{i}}\left(a n n_{R_{i}}\left(m_{i}\right)\right)=a n n_{M_{i}}\left(r_{i}\right)$ for some $r_{i} \in$ $R_{i}$ which shows that $M_{i}$ is a wq-regular $R_{i}$-module.

\section{Trivial extension of weakly quasi regular modules}

This section deals with trivial extension (idealization) of wq-regular modules. The trivial extension $R \propto M=R \oplus M$ of an $R$-module $M$ is a commutative ring with componentwise addition and multiplication $(a, m)\left(b, m^{\prime}\right)=\left(a b, a m^{\prime}+b m\right)$ for any $a, b \in R ; m, m^{\prime} \in$ $M$ [13]. Also the nilradical of $R \propto M$ is characterized as

$$
\sqrt{0_{R \propto M}}=\sqrt{0} \propto M
$$

in [1] and [6]. So one can easily see that $R \propto M$ is reduced if and only if $R$ is reduced and $M=0$ and hence $R \propto M \cong R$.

Proposition 3.1. $R \propto M$ is a quasi regular ring if and only if $M=0$ and $R$ is a quasi regular ring.

Proof. Follows from the fact that all quasi regular rings are reduced rings.

Proposition 3.2. (i) Let $R \propto M$ be a wq-regular ring. Then $M$ is a wq-regular module.

(ii) Let $M$ be a non-torsion module in which ann $_{M}(I)$ is an m-submodule for all ideals $I$ of $R$. If $R \propto M$ is a wq-regular ring, then $R$ is a wq-regular ring.

Proof. (i) Take an element $m \in M$. Since $R \propto M$ is wq-regular, we can conclude $\operatorname{ann}(\operatorname{ann}(0, m))=\operatorname{ann}\left(r, m^{\prime}\right)$ for some $r \in R, m^{\prime} \in M$. This yields $(0, m)\left(r, m^{\prime}\right)=$ $(0, r m)=(0,0)$, and so $r \in \operatorname{ann}_{R}(m)$. This yields that $a n n_{M}\left(a n n_{R}(m)\right) \subseteq \operatorname{ann}_{M}(r)$. Let $n \in \operatorname{ann}_{M}(r)$. Then we have $r n=0$ and thereby $\left(r, m^{\prime}\right)(0, n)=(0,0)$, that is, $(0, n) \in$ $\operatorname{ann}\left(r, m^{\prime}\right)=\operatorname{ann}(\operatorname{ann}(0, m))$. Also note that $\operatorname{ann}(0, m)=\operatorname{ann}_{R}(m) \propto M$. Then we have $(0, n) \in \operatorname{ann}\left(\operatorname{ann}_{R}(m) \propto M\right)$, and so $\operatorname{ann}_{R}(m) n=0$. This gives $n \in \operatorname{ann}_{M}\left(\operatorname{ann}_{R}(m)\right)$. Hence we have $a n n_{M}\left(a n n_{R}(m)\right)=a n n_{M}(r)$, i.e, $M$ is a wq-regular module.

(ii) Let $a \in R$. Then $\operatorname{ann}(a, 0)=\left\{\left(r, m^{\prime}\right):(a, 0)\left(r, m^{\prime}\right)=\left(a r, a m^{\prime}\right)=(0,0)\right\}=$ $\operatorname{ann}_{R}(a) \propto \operatorname{ann}_{M}(a)$. Then $\left(s, m^{\prime}\right) \in \operatorname{ann}(\operatorname{ann}(a, 0))$ if and only if $\left(s, m^{\prime}\right) \in \operatorname{ann}\left(a n n_{R}(a) \propto\right.$ $\left.\left.\operatorname{ann}_{M}(a)\right)\right)$ if and only if $\operatorname{sann}_{R}(a)=0$ and $\operatorname{sann}_{M}(a)+a n n_{R}(a) m^{\prime}=0$. As $M$ is nontorsion, we can conclude $\left(a n n_{M}(a): M\right)=\operatorname{ann}_{R}(a)$, and so $\operatorname{sann}_{R}(a)=0$ implies that $s\left(a n n_{M}(a): M\right)=0$. Thus by assumption, we also get $\operatorname{sann}_{M}(a)=0$. Then we get $a n n_{R}(a) m^{\prime}=0$ and note that

$$
\operatorname{ann}(\operatorname{ann}(a, 0))=\operatorname{ann}_{R}\left(a n n_{R}(a)\right) \propto \operatorname{ann}_{M}\left(a n n_{R}(a)\right) .
$$

Since $R \propto M$ is wq-regular, we have $\operatorname{ann}(\operatorname{ann}(a, 0))=\operatorname{ann}(s, m)$ for some $s \in R, m \in$ $M$. Thus we get $(a, 0)(s, m)=(s a, a m)=(0,0)$. This yields that $s \in$ ann $(a)$, and so $\operatorname{ann}_{R}\left(\operatorname{ann}_{R}(a)\right) \subseteq \operatorname{ann}_{R}(s)$. Now take $t \in \operatorname{ann}_{R}(s)$. Then $s t=0$. Now choose $m^{*} \in$ $M-T(M)$. Then note that $(s, m)\left(0, t m^{*}\right)=(0,0)$, and so $\left(0, t m^{*}\right) \in \operatorname{ann}(s, m)$. This yields that $t m^{*} \in \operatorname{ann}_{M}\left(a n n_{R}(a)\right)$, and so $a n n_{R}(a) t m^{*}=0$. Therefore we conclude that $a n n_{R}(a) t=0$, and so $t \in a n n_{R}\left(a n n_{R}(a)\right)$. Hence we get $a n n_{R}\left(a n n_{R}(a)\right)=a n n_{R}(s)$, that is, $R$ is a wq-regular ring. 
Proposition 3.3. Let $R$ be a wq-regular ring and let $M$ be a non-torsion reduced module satisfying the condition ann $_{R}(m)=$ ann $_{R}(r)$. Further assume that ann $n_{M}(I)$ is an $m$ submodule of $M$ for each ideal $I$ of $R$. Then $R \propto M$ is a wq-regular ring.

Proof. Let $(r, m) \in R \propto M$. Then note that $\left(s, m^{\prime}\right) \in \operatorname{ann}(r, m)$ implies that $s r=0$ and $s m+r m^{\prime}=0$. So we conclude that $s\left(s m+r m^{\prime}\right)=s^{2} m=0$. Since $M$ is reduced, we can conclude $s m=0$, and hence $r m^{\prime}=0$. Thus we deduce

$$
\operatorname{ann}(r, m)=\left(\operatorname{ann}_{R}(r) \cap \operatorname{ann}_{R}(m)\right) \propto \operatorname{ann}_{M}(r) .
$$

Since $R$ is quasi-regular, by assumption we have $a n n_{R}(m)=a n n_{R}(a)$ and so $a n n_{R}(r) \cap$ $a n n_{R}(a)=a n n_{R}(b)$ for some $b \in R$ by [5, Theorem 3.4]. So $\operatorname{ann}(r, m)=a n n_{R}(b) \propto$ $\operatorname{ann}_{M}(r)$. Then $\left(s, m^{\prime}\right) \in \operatorname{ann}(\operatorname{ann}(r, m))$ implies that $\operatorname{sann}_{R}(b)=0$ and $\operatorname{sann}_{M}(r)+$ $a n n_{R}(b) m^{\prime}=0$. Thus we conclude that $s\left(\operatorname{sann}_{M}(r)+a n n_{R}(b) m^{\prime}\right)=0$, and so $s^{2} a n n_{M}(r)=$ 0 . Since $M$ is a reduced module, $\operatorname{sann}_{M}(r)=0$, and thus $a n n_{R}(b) m^{\prime}=0$. So it follows that

$$
\operatorname{ann}(\operatorname{ann}(r, m))=\left(\operatorname{ann}_{R}\left(a n n_{R}(b)\right) \cap a n n_{R}\left(a n n_{M}(r)\right)\right) \propto a n n_{M}\left(a n n_{R}(b)\right) .
$$

By assumption, $t \in \operatorname{ann}_{R}\left(a n n_{M}(r)\right)$ if and only if $t\left(a n n_{M}(r)\right)=t\left(a n n_{M}(r): M\right) M=$ $t\left(a n n_{R}(r)\right) M=0$ if and only if $t \in a n n_{R}\left(a n n_{R}(r)\right)$. Since $R$ is quasi-regular, $a n n_{R}\left(a n n_{R}(b)\right)=$ $a n n_{R}(x)$ and also $a n n_{R}\left(a n n_{R}(r)\right)=a n n_{R}(y)$ for some $x, y \in R$. Also note that $\operatorname{ann}_{M}\left(\operatorname{ann}_{R}(b)\right)=\operatorname{ann}_{M}(x)$. Now choose $m^{*} \in M-T(M)$. Then we have $a n n_{R}(y)=$ $a n n_{R}\left(y m^{*}\right)$, and so

$$
\begin{aligned}
\operatorname{ann}(\operatorname{ann}(r, m)) & =\left(\operatorname{ann}_{R}(x) \cap \operatorname{ann}_{R}\left(y m^{*}\right)\right) \propto \operatorname{ann}_{M}(x) \\
& =\operatorname{ann}\left(x, y m^{*}\right) .
\end{aligned}
$$

Accordingly, $R \propto M$ is a wq-regular ring.

Theorem 3.4. Let $M$ be a non-torsion reduced module in which ann $n_{M}(I)$ is an $m$ submodule of $M$ for all ideals $I$ of $R$. Then $R \propto M$ is a wq-regular ring if and only if $M$ is a wq-regular module.

Proof. It can be obtained from Proposition 3.3 and Proposition 3.2.

\section{Extension of weakly quasi regular modules}

In this section, we study polynomial modules and power series modules. Let $M$ be an $R$-module and let $M[X]$ denote the set of all polynomials in indeterminate $X$ with coefficients in $R$. Then $M[X]$ becomes an $R[X]$-module. Note that if $M$ is a reduced module, then for any $m(X)=m_{0}+m_{1} X+\ldots+m_{n} X^{n} \in M[X]$, where $m_{i} \in M$,

$$
\operatorname{ann}_{R[X]}(m(x))=\left[\bigcap_{i=0}^{n} a n n_{R}\left(m_{i}\right)\right][X] .
$$

Proposition 4.1. Assume $M$ is a reduced non-torsion wq-regular module. Then $M[X]$ is a wq-regular $R[X]$ module.

Proof. Let $m(X)=m_{0}+m_{1} X+\ldots+m_{n} X^{n} \in M[X]$. Since $M$ is reduced, we have $\operatorname{ann}_{R[X]}(m(x))=\left[\bigcap_{i=0}^{n} a n n_{R}\left(m_{i}\right)\right][X]$. As $M$ is a non-torsion reduced module, $R$ is a reduced ring. To see this, take an element $a^{2}=0$. As $M$ is a non torsion module, there is an $m^{*} \in M$ with $\operatorname{ann}_{R}\left(m^{*}\right)=0$. Then note that $a^{2} m^{*}=0$. As $M$ is a reduced module, we get $a m^{*}=0$, and thus $a=0$. As $M$ is a non-torsion wq-regular module over a reduced ring $R$, by Lemma $2.18, M$ satisfies annihilator condition, so that $\bigcap_{i=0}^{n} a n n_{R}\left(m_{i}\right)=a n n_{R}\left(m^{\prime}\right)$ for 
some $m^{\prime} \in M$. Thus $a n n_{R[X]}(m(X))=\left(a n n_{R}\left(m^{\prime}\right)\right)[X]$. Also it can be easily verified that $\operatorname{ann}_{M[X]}(I[X])=\left(\operatorname{ann}_{M}(I)\right)[X]$ for any ideal $I$ of $R$. Then we conclude that

$$
\begin{aligned}
\operatorname{ann}_{M[X]}\left(\operatorname{ann}_{R[X]}(m(X))\right. & =\operatorname{ann}_{M[X]}\left(\left(\operatorname{ann}_{R}\left(m^{\prime}\right)\right)[X]\right) \\
& =\left[\operatorname{ann}_{M}\left(\operatorname{ann}_{R}\left(m^{\prime}\right)\right)\right][X] .
\end{aligned}
$$

Since $M$ is a quasi regular module, there is an $a \in R$ so that $a n n_{M}\left(a n n_{R}\left(m^{\prime}\right)\right)=a n n_{M}(a)$, and so

$$
\operatorname{ann}_{M[X]}\left(\operatorname{ann}_{R[X]}(m(X))=\left(a n n_{M}(a)\right)[X] .\right.
$$

Put $r(X)=a \in R[X]$. Then we have

$$
\operatorname{ann}_{M[X]}\left(a n n_{R[X]}(m(X))=\operatorname{ann}_{M[X]}(r(X)) .\right.
$$

Hence $M[X]$ is a wq-regular $R[X]$ module.

Proposition 4.2. Assume $M$ is a reduced non-torsion $R$-module in which ann $n_{M}(I)$ is an $m$-submodule for each ideal $I$ of $R$. Further assume that $M$ satisfies annihilator condition and for each $m \in M$, ann $_{R}(m)=\operatorname{ann}_{R}(r)$ for some $r \in R$. If $M[X]$ is a wq-regular $R[X]$ module, then $M$ is a wq-regular $R$-module.

Proof. Let $m \in M$. Put $m(X)=m \in M[X]$. As $M[X]$ is a wq-regular $R[X]$ module, we can conclude

$$
\operatorname{ann}_{M[X]}\left(\operatorname{ann}_{R[X]}(m(X))=\left[\operatorname{ann}_{M}\left(a n n_{R}(m)\right)\right][X]=\operatorname{ann}_{M[X]}(r(X)),\right.
$$

where $r(X)=r_{0}+r_{1} X+\ldots+r_{k} X^{k}, r_{i} \in R$. Note that

$$
\operatorname{ann}_{M[X]}(r(X))=\left[\bigcap_{i=0}^{k} a n n_{M}\left(r_{i}\right)\right][X] .
$$

Now we will show that for any $a, b \in R$ there is $c \in R$ such that

$$
\operatorname{ann}_{M}(a) \cap \operatorname{ann}_{M}(b)=\operatorname{ann}_{M}(c) .
$$

Since $M$ is non-torsion, we have $\operatorname{ann}\left(m^{*}\right)=0$ for some $m^{*} \in M$, and so $\operatorname{ann}_{R}(a)=$ $a n n_{R}\left(a m^{*}\right), a n n_{R}(b)=a n n_{R}\left(b m^{*}\right)$. By annihilator condition, $a n n_{R}\left(a m^{*}\right) \cap a n n_{R}\left(b m^{*}\right)=$ $\operatorname{ann}_{R}\left(m^{\prime}\right)$ for some $m^{\prime} \in M$. By assumption, there is an $c \in R$ so that $a n n_{R}\left(m^{\prime}\right)=$ $\operatorname{ann}_{R}(c)$. Since $M$ is non-torsion,

$$
\begin{aligned}
\left(\operatorname{ann}_{M}(R a+R b)\right. & \left.:_{R} M\right)=\operatorname{ann}_{R}(R a+R b) \\
& =\operatorname{ann}_{R}(a) \cap a n n_{R}(b) \\
& =\operatorname{ann}_{R}\left(a m^{*}\right) \cap a n n_{R}\left(b m^{*}\right) \\
& =\operatorname{ann}_{R}(c)=\left(\operatorname{ann}_{M}(c):_{R} M\right) .
\end{aligned}
$$

This implies that

$$
\begin{aligned}
\left(\operatorname{ann}_{M}(R a+R b)\right. & \left.:_{R} M\right) M=\operatorname{ann}_{M}(R a+R b) \\
& =\operatorname{ann}_{M}(a) \cap \operatorname{ann}_{M}(b) \\
& =\left(\operatorname{ann}_{M}(c):_{R} M\right) M \\
& =\operatorname{ann}_{M}(c) .
\end{aligned}
$$

Then for $r_{0}, r_{1}, \ldots, r_{k} \in R, \bigcap_{i=0}^{k} a n n_{M}\left(r_{i}\right)=a n n_{M}(y)$ for some $y \in R$. This yields

$$
\begin{aligned}
\operatorname{ann}_{M[X]}\left(\operatorname{ann}_{R[X]}(m(X))\right. & =\left[\operatorname{ann}_{M}\left(\operatorname{ann}_{R}(m)\right)\right][X] \\
& =\left(\operatorname{ann}_{M}(y)\right)[X] .
\end{aligned}
$$

Thus we have $a n n_{M}\left(a n n_{R}(m)\right)=a n n_{M}(y)$. Accordingly, $M$ is a wq-regular $R$-module. 
Let $M$ be an $R$-module and let $M[[X]]$ denote the formal power series module over $R[[X]]$.

Definition 4.3. An $R$-module $M$ is said to satisfy the countably annihilator condition if for each family of $\left\{m_{n}\right\}_{n \in \mathbb{N}}$, then $\bigcap_{i=1}^{\infty} a n n_{R}\left(m_{i}\right)=a n n_{R}(m)$ for some $m \in M$.

Proposition 4.4. Assume $M$ is a reduced wq-regular module satisfying the countably annihilator condition. Then $M[[X]]$ is a wq-regular $R[[X]]$-module.

Proof. Let $f(X)=\sum_{i=0}^{\infty} m_{i} X^{i} \in M[[X]]$. As $M$ is a reduced module, ann $R[[X]](f(X))=$ $\left(\bigcap_{i=0}^{\infty} \operatorname{ann}_{R}\left(m_{i}\right)\right)[[X]]$. As $M$ satisfies the countably annihilator condition, $\operatorname{ann}_{R[[X]]}(f(X))=$ $\left(\operatorname{ann}_{R}(m)\right)[[X]]$ for some $m \in M$. This yields

$$
a \operatorname{ann_{M[[X]]}}\left(a n n_{R[[X]]}(f(X))\right)=\operatorname{ann}_{M[[X]]}\left(\left(a n n_{R}(m)\right)[[X]]\right) .
$$

It is obvious that $a n n_{M[[X]]}\left(\left(a n n_{R}(m)\right)[[X]]\right)=\left(a n n_{M}\left(a n n_{R}(m)\right)[[X]]\right.$. As $M$ is wqregular, $\operatorname{ann}_{M}\left(a n n_{R}(m)\right)=a n n_{M}(a)$ for some $a \in R$. Thus

$$
a \operatorname{ann} n_{M[[X]]}\left(a n n_{R[[X]]}(f(X))\right)=\left(a n n_{M}(a)\right)[[X]] .
$$

Now put $g(X)=a \in R[[X]]$ and note that $\left(\operatorname{ann}_{M}(a)\right)[[X]]=\operatorname{ann}_{M[[X]]}(g(X))$. Accordingly, $M[[X]]$ is a wq-regular $R[[X]]$-module.

Proposition 4.5. Assume $M$ is a reduced non-torsion $R$-module in which ann $n_{M}(I)$ is an $m$-submodule for each ideal $I$ of $R$. Further, suppose $M$ satisfies the countably annihilator condition and for each $m \in M$, ann $n_{R}(m)=$ ann $_{R}(r)$ for some $r \in R$. If $M[[X]]$ is a wq-regular $R[[X]]$-module, then $M$ is a wq-regular $R$-module.

Proof. Let $m \in M$. Put $f(X)=m \in M[[X]]$. Then $\operatorname{ann}_{M[[X]]}\left(a n n_{R[[X]]}(f(X))\right)=$ $\operatorname{ann}_{M[[X]]}(g(X))$ for some $g(X)=\sum_{i=0}^{\infty} a_{i} X^{i}$, where $a_{i} \in R$. This implies that

$$
\left(\operatorname{ann}_{M}\left(\operatorname{ann}_{R}(m)\right)\right)[[X]]=\left(\bigcap_{i=0}^{\infty} \operatorname{ann}_{M}\left(a_{i}\right)\right)[[X]]
$$

As $M$ is non-torsion, we get $m^{*} \in M-T(M)$. Then $\bigcap_{i=0}^{\infty} a n n_{R}\left(a_{i} m^{*}\right)=a n n_{R}\left(m^{\prime}\right)$ for some $m^{\prime} \in M$ by the countably annihilator condition. By assumption, there is $b \in R$ so that $a n n_{R}\left(m^{\prime}\right)=a n n_{R}(b)$, and so

$$
\begin{aligned}
\left(\operatorname{ann}_{M}\left(\sum_{i=0}^{\infty} R a_{i}\right)\right. & \left.:_{R} M\right)=\operatorname{ann} n_{R}\left(\sum_{i=0}^{\infty} R a_{i}\right) \\
& =a n n_{R}\left(\sum_{i=0}^{\infty} R a_{i} m^{*}\right)=a n n_{R}\left(m^{\prime}\right) \\
& =\operatorname{ann}_{R}(b)=\left(\operatorname{ann}_{M}(b):_{R} M\right) .
\end{aligned}
$$

Then

$$
\begin{aligned}
\left(\operatorname{ann}_{M}\left(\sum_{i=0}^{\infty} R a_{i}\right)\right. & \left.:_{R} M\right) M=\bigcap_{i=0}^{\infty} \operatorname{ann}_{M}\left(a_{i}\right) \\
& =\left(\operatorname{ann}_{M}(b):_{R} M\right) M=\operatorname{ann}_{M}(b) .
\end{aligned}
$$

This implies that

$$
\begin{aligned}
\operatorname{ann}_{M[[X]]}\left(\operatorname{ann}_{R[[X]]}(f(X))\right) & =\left(\operatorname{ann}_{M}\left(a n n_{R}(m)\right)\right)[[X]] \\
& =\left(\operatorname{ann}_{M}(b)\right)[[X]],
\end{aligned}
$$

and so $a n n_{M}\left(a n n_{R}(m)\right)=a n n_{M}(b)$. This gives that $M$ is a wq-regular $R$-module. 
Theorem 4.6. Let $M$ be a reduced non-torsion module in which ann $n_{M}(I)$ is an $m$ submodule for each ideal $I$ of $R$. Assume $M$ satisfies the countably annihilator condition and for each $m \in M$, ann $(m)=a n n_{R}(r)$ for some $r \in R$. Then the following are equivalent:

(i) $M$ is a wq-regular $R$-module.

(ii) $M[X]$ is a wq-regular $R[X]$-module.

(iii) $M[[X]]$ is a wq-regular $R[[X]]$-module.

Proof. $(i) \Leftrightarrow($ ii) It can be obtained from Proposition 4.1 and Proposition 4.2.

$(i) \Leftrightarrow($ iii $)$ It can be obtained from Proposition 4.4 and Proposition 4.5.

Acknowledgment. We would like to thank the referee for his/her great effort in proofreading the manuscript.

\section{References}

[1] D.D. Anderson and M. Winders, Idealization of a module, J. Commut. Algebra, 1 (1), 3-56, 2009.

[2] D.F. Anderson, R. Levy and J. Shapiro, Zero-divisor graphs, von Neumann regular rings, and Boolean algebras, J. Pure Appl. Algebra, 180 (3), 221-241, 2003.

[3] Z.A. El-Bast and P.F. Smith, Multiplication modules, Comm. Algebra, 16 (4), 755$779,1988$.

[4] M. Evans, On commutative P.P. rings, Pac. J. Math. 41 (3), 687-697, 1972

[5] M. Henriksen and M. Jerison, The space of minimal prime ideals of a commutative ring, Trans. Amer. Math. Soc. 115, 110-130, 1965.

[6] J.A. Huckaba, Commutative rings with zero divisors, Marcel Dekker, New York, 1988.

[7] C. Jayaram, Baer ideals in commutative semiprime rings, Indian J. Pure Appl. Math. 15 (8) 855-864, 1984.

[8] C. Jayaram and Ü. Tekir, von Neumann regular modules, Comm. Algebra, 46 (5), 2205-2217, 2018.

[9] T.K. Lee and Y. Zhou, Reduced modules, Rings, modules, algebras and abelian groups, in:Lect. Notes Pure Appl. Math. New York, NY: Marcel Dekker, 236, 365-377, 2004.

[10] R. Levy and J. Shapiro, The zero-divisor graph of von Neumann regular rings, Comm. Algebra, 30 (2), 745-750, 2002.

[11] C.P. Lu, Prime submodules of modules, Comment. Math. Univ. St. Pauli, 33 (1), 61-69, 1984.

[12] R.L. McCasland and M.E. Moore, Prime submodules, Comm. Algebra, 20 (6), 18031817, 1992.

[13] M. Nagata, Local rings, Interscience Publishers, New York, 1960.

[14] J. Von Neumann, On regular rings, Proc. Natl. Acad. Sci. 22 (12), 707-713, 1936. 